\title{
An Approach for Improvement of the Dissolution Rate of Gliclazide
}

\author{
Sudarsan Biswal', Gouri S. Pasa, Jagannath Sahoo, \\ and Padala N. Murthy \\ Royal College of Pharmacy and Health Sciences, Andhapasara Road, Berhampur-760002, \\ Orissa, India
}

e-mail: sudarsan_mpharm@yahoo.co.in

\begin{abstract}
Gliclazide is an anti-diabetic drug that is poorly soluble in water. This paper describes an approach to improve the dissolution rate of gliclazide by using solid dispersions (SDs) in polyethylene glycol 4000 (PEG 4000). The phase-solubility behavior of gliclazide in the presence of various concentrations of PEG 4000 in $0.1 \mathrm{~N} \mathrm{HCl}$ at $37{ }^{\circ} \mathrm{C}$ was obtained. The solubility of gliclazide increased with increasing amounts of PEG 4000 in water. The Gibbs free energy $\left(\Delta G^{\circ}{ }_{\text {tr }}\right)$ values were all negative. The solid dispersions were prepared with a solvent-melting method using different concentrations of PEG 4000. X-ray diffraction, infrared spectroscopy, and DSC were used to examine the physicochemical characteristics of solid dispersions of gliclazide and PEG. The dissolution rate of gliclazide in SDs with PEG 4000 was enhanced. The FTIR spectroscopic studies showed the presence of intermolecular hydrogen bonding between gliclazide and PEG 4000 in the solid state. The DSC and XRD studies indicate the amorphous and microcrystalline states of gliclazide in SDs with PEG 4000.
\end{abstract}

\section{INTRODUCTION}

G liclazide is a second-generation hypoglycemic sulfonylurea that is useful in the treatment of Type 2 diabetes mellitus (1). Gliclazide shows good tolerability and a low incidence of hypoglycemia; a low rate of secondary failure inhibits platelet aggregation and increases fibrinolysis $(2,3)$. Thus gliclazide appears to be a drug of choice in long-term sulfonylurea therapy for the control of Type 2 diabetes mellitus (4). It shows low aqueous solubility and dissolution rate and often shows low and irregular bioavailability after oral administration. The enhancement of oral bioavailability of poorly watersoluble drugs remains one of the most challenging aspects of formulation development. The solid dispersion of poorly water-soluble drugs in water-soluble polymers enhances drug dissolution and bioavailability (5).

The preparation and characterization of complexes of gliclazide with $\beta$-cyclodextrin have been reported (6-8). Complexation of gliclazide with $\beta$-cyclodextrinhydroxypropyl methylcellulose, which enhanced its hypoglycemic activity, has been reported (9). In addition, accelerated absorption of gliclazide using PEG 400 was studied earlier (10). Solid dispersions of gliclazide in PEG 6000 have been developed to increase drug dissolution rate (11). Enhancement of the solubility of gliclazide using polyvinylpyrrolidone $\mathrm{K} 90$ has been reported (12). The molecular weight of the polymer may play a role in the performance of a solid dispersion. The rationale of the present study was to investigate the use of lower molecular weight PEG 4000 for the preparation of solid dispersions with the objectives of improving dissolution

${ }^{1}$ Corresponding author. rate of gliclazide and obtaining different behavior as compared with PEG 6000.

\section{MATERIALS AND METHODS \\ Materials}

A gift sample of gliclazide was received from Aristo Pharmaceuticals Ltd. (Mumbai, India). PEG 4000 was received from Clariant (Germany). Double-distilled water was used throughout the study, and all other chemicals were of analytical grade.

\section{Preparation of SDs}

The SDs of gliclazide with PEG 4000 contained three different weight ratios of gliclazide/PEG 4000 (1:1, 1:2, 1:5), which are denoted as SD 1/1, SD 1/2, and SD 1/5, respectively. The solid dispersions were prepared by a solvent-melting method using different concentrations of PEG 4000. In the solvent-melting method, the required amount of PEG 4000 was melted in a glass container on a water bath maintained at a temperature of about 50$65^{\circ} \mathrm{C}$. The required amount of gliclazide solution in chloroform was added to the molten PEG 4000 and mixed thoroughly with a glass rod for $5 \mathrm{~min}$. The glass container was placed in an ice bath for about $5 \mathrm{~min}$; the mixture cooled rapidly and solidified. The hardened mixture was then powdered in a mortar, sieved through a 100 -mesh screen, and stored in a screw-cap vial at room temperature until use.

Physical mixtures (PMs) having the same weight ratios as the SDs were prepared by thoroughly mixing the required amounts of gliclazide and PEG 4000 for 10 min in a mortar. The resulting mixtures were sieved through a 100-mesh sieve and denoted as PM 1/1, PM 1/2, and PM $1 / 5$. The mixtures were stored in screw-cap vials at room temperature until use. 


\section{Solubility Determinations of Gliclazide}

Solubility determinations were performed in triplicate according to the method of Higuchi and Connors (13). An excess amount of gliclazide was placed in a screw-cap glass vial to which $20 \mathrm{~mL}$ of an aqueous solution containing various concentrations of PEG 4000 was added. The samples were shaken at $37 \pm 0.5^{\circ} \mathrm{C}$ for $72 \mathrm{~h}$ in a water bath (Remi Pvt Ltd, Mumbai). After 72 h, the samples were filtered through a $0.45-\mu \mathrm{m}$ membrane filter. The filtrate was suitably diluted and analyzed spectrophotometrically at $227 \mathrm{~nm}$ using a UV-vis spectrophotometer (Shimadzu UV-1700, Pharm Spec).

\section{Dissolution Studies}

Dissolution studies of gliclazide in powder form, SDs, and PMs were performed using USP Apparatus 2 (Lab India, Mumbai) at a paddle rotation speed of $50 \mathrm{rpm}$ in $900 \mathrm{~mL} 0.1 \mathrm{~N} \mathrm{HCl}$ containing $0.25 \%(\mathrm{w} / \mathrm{v}) \mathrm{SLS}$ at $37 \pm$ $0.5^{\circ} \mathrm{C}$. SDs or PMs equivalent to $30 \mathrm{mg}$ of gliclazide were weighed using a digital balance (Sartorius) and added to the dissolution medium. At specified times (every $10 \mathrm{~min}$ for $1 \mathrm{~h}$ ), 10-mL samples were withdrawn using a $0.45-\mu \mathrm{m}$ syringe filter (Sepyrane, Mumbai) and then assayed for the gliclazide content by measuring the absorbance at $227 \mathrm{~nm}$ using a UV-vis spectrophotometer (Shimadzu UV-1700, PharmSpec). Fresh medium (10 mL) that was prewarmed at $37^{\circ} \mathrm{C}$ was added to the dissolution medium after each sampling to maintain a constant volume throughout the test. Dissolution studies were performed in triplicate $(n=3)$, and calculated mean values of cumulative drug release were used to plot the release curves.

\section{Fourier Transform Infrared Spectroscopy}

Infrared spectra were obtained using an FTIR-430 spectrometer (Jasco, Japan). Samples of gliclazide, SDs, and PMs were ground and mixed thoroughly with potassium bromide at a 1:5 sample/ $\mathrm{KBr}$ ratio. The $\mathrm{KBr}$ discs were prepared by compressing the powders at a pressure of $5 \mathrm{~T}$ for $5 \mathrm{~min}$ in a hydraulic press. Forty scans from 4000 to $400 \mathrm{~cm}^{-1}$ were obtained at a resolution of $4 \mathrm{~cm}^{-1}$.

\section{Differential Scanning Calorimetry}

The DSC measurements were performed on a DSC-6100 (Seiko Instruments, Japan) differential scanning calorimeter with a thermal analyzer. All accurately weighed samples (about $1.675 \mathrm{mg}$ of gliclazide or equivalent) were placed in sealed aluminum pans before heating under nitrogen flow $(20 \mathrm{~mL} / \mathrm{min})$ at a scanning rate of $10^{\circ} \mathrm{C} / \mathrm{min}$ from 25 to $250^{\circ} \mathrm{C}$. An empty aluminum pan was used as reference.

\section{X-Ray Diffraction}

X-ray powder diffraction patterns were obtained at room temperature using a PW1710 X-ray diffractometer (Philips, Holland) with a Cu anode and a graphite monochromator, operated at a voltage of $35 \mathrm{kV}$ and a current of $20 \mathrm{~mA}$. The samples were analyzed in the $2 \theta$ angle range of $5-70^{\circ}$, and the process parameters were set as scan-step size of $0.02^{\circ}(2 \theta)$ and scan-step time of $0.5 \mathrm{~s}$.

\section{Dissolution Data Analysis}

Phase-Solubility Studies

The value of the apparent stability constant, $K_{s,}$ for drug-carrier combinations was computed from the phase-solubility profiles, as described by

$$
K_{s}=\frac{\text { Slope }}{\text { Intercept }(1-\text { Slope })}
$$

The value of $K_{s}$ for drug-carrier combinations can also be computed from the phase-solubility profiles by

$$
K_{s}=\frac{S_{t}-S_{0}}{S_{0}\left(L_{t}-S_{t}+S_{0}\right)}
$$

where $S_{t}$ is the total concentration of dissolved drug, $S_{0}$ is the equilibrium solubility of drug in the presence of polymer, and $L_{t}$ is the total concentration of polymer (14).

The Gibbs free energy of transfer $\left(\Delta G_{\mathrm{tr}}{ }^{\circ}\right)$ of gliclazide from pure water to aqueous solutions of carrier was calculated as

$$
\Delta G_{\mathrm{tr}}=-2.303 R T\left(\log \frac{S_{0}}{S_{S}}\right)
$$

where $S_{0} / S_{S}$ is the ratio of the molar solubility of gliclazide in aqueous solution of PEG 4000 to that in the same medium without PEG 4000.

\section{RESULTS AND DISCUSSION \\ Solubility Studies}

The phase-solubility diagram investigated in $0.1 \mathrm{~N} \mathrm{HCl}(\mathrm{pH} 1.2)$ is linear over a wide range of PEG 4000 concentrations and corresponds to $\mathrm{A}_{\mathrm{L}}$-type profiles (13). The stability constant is $0.193 \mathrm{mg} / \mathrm{mL}$. The values of the stability constant depend on slope values. The greater the value of the slope, the greater is the capacity of the polymer to solubilize the drug. It has been reported that Gelucire 44/14, which has a larger slope value, shows a greater capacity to solubilize halofantrine than PEG 8000 and PVP K 30 (15). The stability constant values vary slightly with polymer molecular weight. The values of the stability constants increase with increasing polymer molecular weight. Trapani et al. (16) reported that PEG 6000 shows a lower solubilization capacity than PEG 4000 (102 and 92 mg/mL for PEG 6000 and PEG 4000, respectively). These results agree with the well-established formation of soluble complexes between the watersoluble polymeric carriers and poorly water-soluble drugs (17). At an $18 \%(w / v)$ concentration of PEG 4000 , the solubility of gliclazide increased by a factor of 3.4 (Table 1). Increased solubility may be due to the improved wettability of the gliclazide particles in aqueous solution 


\begin{tabular}{|c|c|c|}
\hline \multicolumn{3}{|c|}{$\begin{array}{l}\text { Table 1. Effect of PEG } 4000 \text { Concentration and Gibbs Free } \\
\text { Energy on Gliclazide Solubility }\end{array}$} \\
\hline $\begin{array}{l}\text { Concentration of } \\
\text { PEG } \mathbf{4 0 0 0}(\% \mathrm{w} / \mathrm{v})\end{array}$ & $\begin{array}{l}\text { Concentration of Gliclazide } \\
(\mathrm{mg} / \mathrm{mL}) \text { at } 37^{\circ} \mathrm{C}\end{array}$ & $\begin{aligned} \Delta \boldsymbol{G}_{\mathrm{tr}}^{\circ} \\
(\mathrm{J} / \mathrm{Mol}\end{aligned}$ \\
\hline 0 & $0.80 \pm 0.02$ & 0 \\
\hline 2 & $0.81 \pm 0.01$ & 0 \\
\hline 4 & $1.06 \pm 0.05$ & -698 \\
\hline 6 & $1.28 \pm 0.01$ & -1184 \\
\hline 8 & $1.53 \pm 0.05$ & -1644 \\
\hline 10 & $1.76 \pm 0.08$ & -2005 \\
\hline 12 & $1.97 \pm 0.09$ & -2295 \\
\hline 14 & $2.2 \pm 0.01$ & -2580 \\
\hline 16 & $2.45 \pm 0.02$ & -2857 \\
\hline 18 & $2.75 \pm 0.08$ & -3155 \\
\hline
\end{tabular}

from PEG 4000. The values of Gibbs free energy change are an indication of the process of transfer of gliclazide from pure water to aqueous solution of PEG 4000. Table 1 presents the values of the Gibbs free energy associated with the aqueous solubility of gliclazide in the presence of PEG 4000. The $\Delta G_{\mathrm{tr}}{ }^{\circ}$ values are all negative for PEG 4000 at various concentrations, indicating the spontaneous nature of the drug solubilization.

\section{Dissolution Studies}

The $Q_{10}, Q_{20}$, and $Q_{30}$ values are reported in Table 2 , and dissolution profiles are shown in Figure 1. From the results in Table 2, it is evident that the dissolution onset of pure gliclazide is very low, about $40.82 \%$ of the drug being dissolved within 30 min. SDs of gliclazide with PEG 4000 show considerably enhanced dissolution rates within 30 min compared with those for pure gliclazide and PMs. Model-independent approaches are based on the ratio of the area under the dissolution curve (dissolution efficiency) or on mean dissolution time $(18,19)$. The percent dissolution efficiency $(\% D E)$ and mean dissolution time (MDT) were also computed to compare the relative performance of various concentrations of carrier in SDs and PMs. The $\% D E$ of a pharmaceutical dosage form is defined as the area under the dissolution curve up to a certain time, $t$, expressed as a percentage of the of the area of the rectangle described by $100 \%$ dissolution at the same time. The $\% D E$ can be calculated from

$$
\% D E=\frac{\int_{0}^{t} Y \mathrm{~d} t}{Y_{100} t} \times 100 \%
$$

where $Y$ is the percent drug dissolved at time $t$.

The value of $\% D E_{10 \mathrm{~min}}$ for pure gliclazide $(9.16 \%)$ was enhanced in PMs (15.45\%) as well as in SDs (35.92\%). The
Table 2. In Vitro Dissolution Profile of Gliclazide, Physical Mixture of Gliclazide, and Solid Dispersion of Gliclazide in $0.1 \mathrm{~N} \mathrm{HCl}(p H$ 1.2)

\begin{tabular}{lcccccc}
\hline & \multicolumn{5}{c}{ Dissolution Parameters } \\
\cline { 2 - 7 } Formulation & $\boldsymbol{Q}_{10}$ & $\boldsymbol{Q}_{\mathbf{2 0}}$ & $\boldsymbol{Q}_{\mathbf{3 0}}$ & $\% \mathrm{DE}_{10 \mathrm{~min}}$ & $\% \mathrm{DE}_{\mathbf{3 0} \text { min }}$ & $\begin{array}{c}\text { MDT } \\
(\mathbf{m i n})\end{array}$ \\
\hline Drug & 18.46 & 32.67 & 40.82 & 9.16 & 23.67 & 12.5 \\
\hline $\mathrm{PM} 1 / 1$ & 22.0 & 34.0 & 51.7 & 11.0 & 27.28 & 15.86 \\
\hline $\mathrm{PM} 1 \frac{1}{2}$ & 22.8 & 38.1 & 54.9 & 11.38 & 29.42 & 15.84 \\
\hline $\mathrm{PM} 1 / 5$ & 30.9 & 42.0 & 57.0 & 15.45 & 33.81 & 14.38 \\
\hline $\mathrm{SD} 1 / 1$ & 54.61 & 63.94 & 67.5 & 27.31 & 50.76 & 12.42 \\
\hline $\mathrm{SD} 1 / 2$ & 59.6 & 65.9 & 69.8 & 29.82 & 53.48 & 11.52 \\
\hline $\mathrm{SD} 1 / 5$ & 71.8 & 78.5 & 82.7 & 35.92 & 63.91 & 9.5 \\
\hline
\end{tabular}

value of $\% D E_{30 \mathrm{~min}}$ for the pure drug was increased to $33.81 \%$ in PMs and up to $63.91 \%$ in SDs (Table 2).

To understand the extent of gliclazide dissolution rate enhancement from its SDs and PMs, the dissolution data for pure gliclazide, SDs, and PMs were used to calculate the mean dissolution time (MDT). The mean dissolution time can be calculated by using eq 5 (20).

$$
M D T_{\text {in vitro }}=\frac{\sum_{i=1}^{n} T_{\text {mid }} \Delta M}{\sum_{i=1}^{n} \Delta M}
$$

Here, $i$ is the dissolution sample number, $n$ is the number of dissolution sampling times, $T_{\text {mid }}$ is the midpoint between times $T_{i}$ and $T_{i-1}$, and $\Delta M$ is the amount of gliclazide dissolved $(\mu \mathrm{g})$ between times $T_{i}$ and $T_{i-1}$.

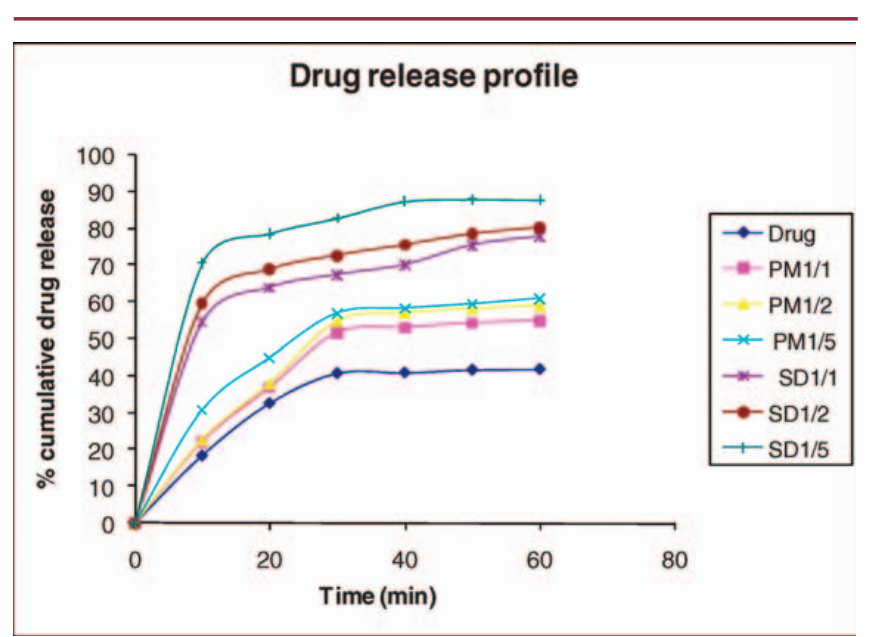

Figure 1. In vitro dissolution profiles of gliclazide, a physical mixture of gliclazide, and a solid dispersion of gliclazide in $0.1 \mathrm{~N} \mathrm{HCl}(\mathrm{pH} 1.2)$.

Dissolution Technologies | NOVEMBER 2009 


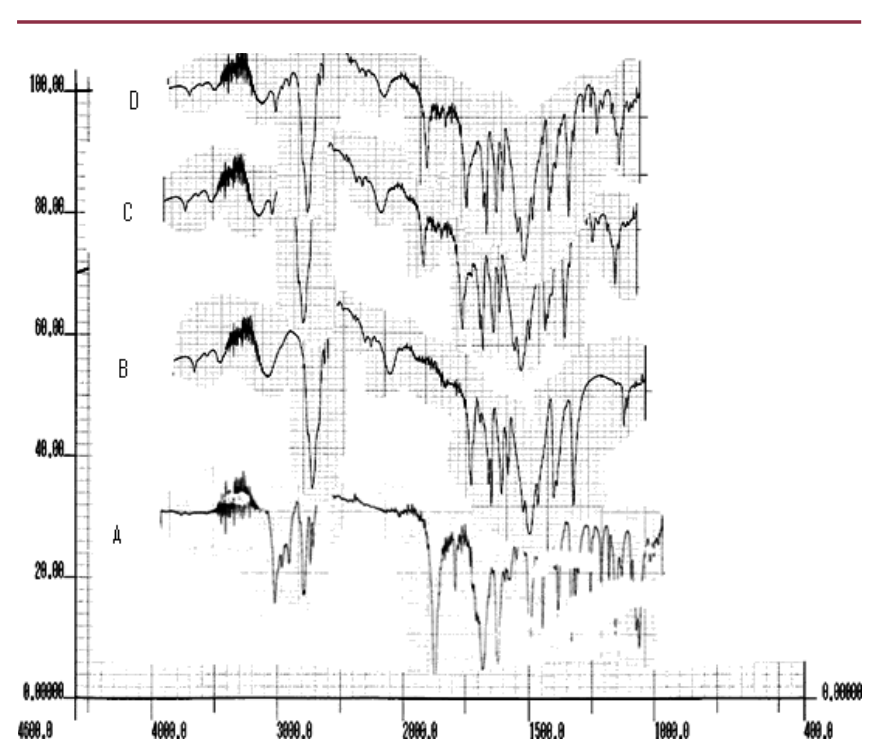

Figure 2. FTIR spectra of (A) pure gliclazide, (B) pure PEG 4000, (C) gliclazide-PEG 4000 PM, and (D) gliclazide-PEG 4000 SD.

The MDT values for pure gliclazide, PMs, and SDs are presented in Table 2. The MDT for gliclazide is $12.5 \mathrm{~min}$; it decreased to $9.5 \mathrm{~min}$ in SDs with PEG 4000 at a 1:5 gliclazide/PEG 4000 ratio.

The magnitude of the mean dissolution time and the percent dissolution efficiency for each formulation were calculated using PCP Disso v3 software (Pune, India).

The increase in dissolution kinetics of gliclazide from PEG SDs may be due to the reduction of crystal size, the absence of aggregation of drug crystals, and the conversion of drug from crystalline to the amorphous or microcrystalline state (5). An increase in gliclazide wettability could result from the formation of a film of polyethylene glycol around it, which modifies the hydrophobicity of the surfaces and explains the mechanism of improved dissolution rate in PMs $(21,22)$.

\section{FTIR Spectroscopy}

FTIR spectroscopy was used to characterize the possible interactions between drug and carrier in the solid state. The IR spectra of SDs and PMs were compared with the standard spectrum of gliclazide (Figure 2A). The IR spectrum of gliclazide is characterized by the absorption of the carbonyl $(C=O)$ sulfonylurea group at $1706 \mathrm{~cm}^{-1}$ (6). In the spectra of the SDs and PMs, this band is shifted toward higher frequencies at $1712 \mathrm{~cm}^{-1}$ and $1720 \mathrm{~cm}^{-1}$, respectively. Also, the $-\mathrm{NH}$ group band at $3265 \mathrm{~cm}^{-1}$ in the spectrum of gliclazide is shifted to $3367 \mathrm{~cm}^{-1}$ in the SDs. The sulfonyl group bands are located at $1349 \mathrm{~cm}^{-1}$ and $1162 \mathrm{~cm}^{-1}$ in pure gliclazide. In the SDs, the asymmetric vibration peak of the $\mathrm{S}=\mathrm{O}$ band is shifted from $1349 \mathrm{~cm}^{-1}$ to $1346 \mathrm{~cm}^{-1}$ with decreased intensity. In the SDs, the symmetrical stretching vibration band of $\mathrm{S}=\mathrm{O}$ is shifted from $1162 \mathrm{~cm}^{-1}$ to $1112 \mathrm{~cm}^{-1}$ with

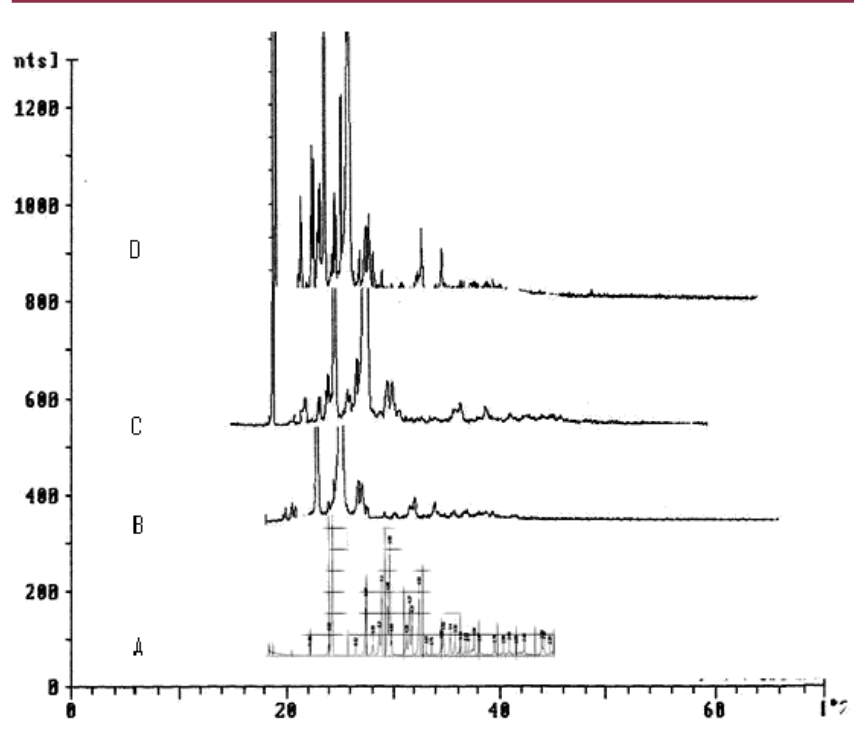

Figure 3. X-Ray diffractograms of (A) pure gliclazide, (B) pure PEG 4000 (C) gliclazide-PEG 4000 PM, and (D) gliclazide-PEG 4000 SD.

decreased intensities. Important vibrations in the spectrum of PEG 4000 are the $\mathrm{C}-\mathrm{H}$ stretch at $2890 \mathrm{~cm}^{-1}$ and the $\mathrm{C}-\mathrm{O}$ stretch at $1113 \mathrm{~cm}^{-1}$. The shift in the peaks associated with the gliclazide sulfonylurea group indicates an increase in bond strength, possibly due to the stabilizing effect of the hydrogen atoms of PEG 4000 interacting with the oxygen atoms of the sulfonyl group. This led to the conclusion that the changes seen are a result of intermolecular hydrogen bonding between gliclazide and PEG 4000 in the solid state.

\section{X-Ray Diffraction}

The diffraction spectrum of pure gliclazide shows that the drug was of crystalline nature as demonstrated by numerous peaks observed at $2 \theta$ of $10.59,14.98,17.2$, $17.85,18.15,22.07,25.42,26.25,26.75$, and 29.5 (Figure 3A). Pure PEG 4000 shows two peaks with the highest intensity at $2 \theta$ and $d$-spacings of 19.14 and $4.63 \AA$; 22.95 and $3.87 \AA ; 23.28$ and $3.81 \AA$; and 23.49 and $3.78 \AA$. Some changes in gliclazide peak position were observed in PMs as well as SDs. The prominent peaks from pure gliclazide were clearly seen at the same positions in the PMs and SDs, but with decreased intensities. The relative reduction in diffraction intensity of gliclazide in PEG 4000 preparations at these angles suggests that the size of the crystals was reduced to a microcrystal form (23). The positions of PEG 4000 peak patterns in the PMs and SDs are the same and superimposable, which, again, rules out the possibility of a well-defined chemical interaction and new compound formation between these two components. Results of this study imply that gliclazide is present in a partially crystalline or microcrystalline form in the SDs. Valizadeh et al. (24) characterized indomethacin-PEG 6000 SDs prepared by a melting method and concluded 


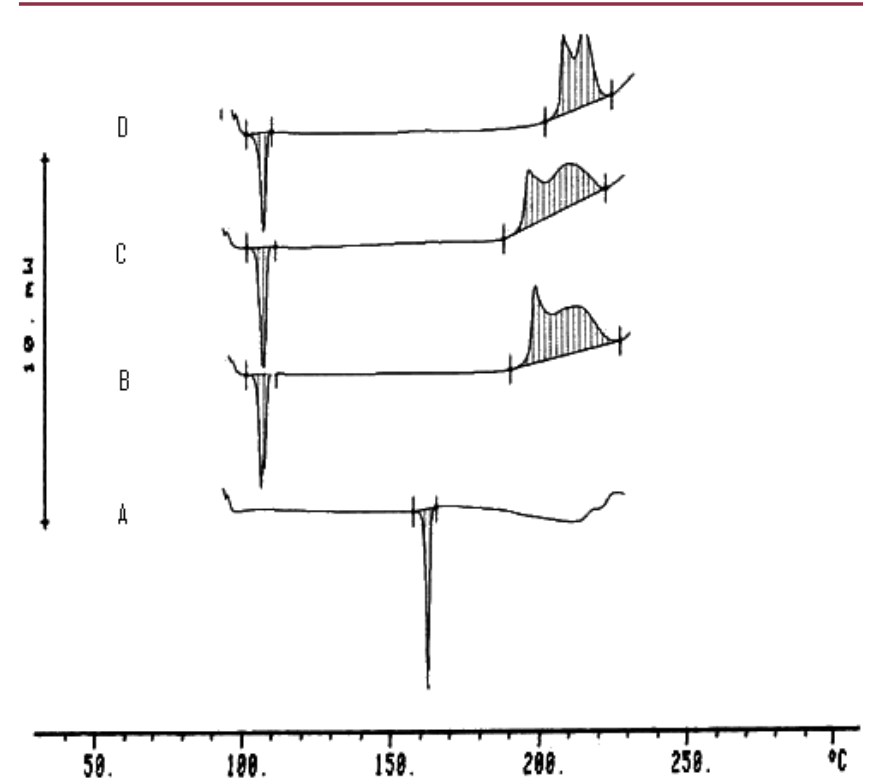

Figure 4. DSC thermograms of (A) pure gliclazide, (B) pure PEG 4000, (C) gliclazide-PEG 4000 PM, and (D) gliclazide-PEG 4000 SD.

that the drug was in a microcrystalline form and that no well-defined chemical interaction took place between indomethacin and PEG 6000, either in solution or in the solid state.

\section{Differential Scanning Calorimetry}

The DSC curve of pure gliclazide exhibits a single endotherm corresponding to the melting of the drug. The onset of melting was observed at $170.8^{\circ} \mathrm{C}$, and the corresponding heat of fusion $\left(\Delta H_{\mathrm{F}}\right)$ is $171.8 \mathrm{~J} / \mathrm{g}$ (Figure 4A), whereas pure PEG 4000 shows a melting endotherm at $60.2^{\circ} \mathrm{C}$ and a corresponding $\Delta H_{\mathrm{F}}$ of $235.0 \mathrm{~J} / \mathrm{g}$. Thermograms of SDs (Figure 4D) show the absence of a gliclazide melting peak and one exothermic peak at $253.9^{\circ} \mathrm{C}$; the corresponding $\Delta H_{\mathrm{F}}$ is $741.6 \mathrm{~J} / \mathrm{g}$ suggesting that gliclazide is completely soluble in the liquid phase of the polymer or that the crystalline nature of gliclazide is absent. The exothermic peak may be due to crystallization above the glass transition temperature, $T_{\mathrm{g}}$. The molecular motion of amorphous solids depends on temperature. The kinetic energy of amorphous solids increases significantly as the temperature increases to $T_{\mathrm{g}}$. Because of the thermodynamic instability of amorphous solids relative to the crystalline state, spontaneous crystallization is always possible as soon as molecular mobility is above the threshold of nucleation. However, the observed melting peak for PEG 4000 in SDs is at the same temperature $\left(59.4^{\circ} \mathrm{C}\right)$ as for pure PEG 4000 . The absence of an endothermic peak of drug in SDs has also been reported by other research groups $(25,26)$. The PM formulations of gliclazide and PEG 4000 also show no melting peak for gliclazide (Figure $4 \mathrm{C}$ ), even though the peaks derived from gliclazide are observed in XRD
(Figure 3A). It is speculated that gliclazide dissolved in the melted PEG 4000 during the DSC measurement; only one endothermic peak at $63.4^{\circ} \mathrm{C}$ corresponding to the melting of PEG 4000 was observed. This result agrees with the report of Yamashita et al. (26); their DSC study reported the absence of an endothermic peak of tacrolimus in the PM formulation of tacrolimus and PEG 4000.

\section{CONCLUSIONS}

The solubility and dissolution rate of gliclazide can be enhanced in SDs with PEG 4000. The solubilization effect of PEG 4000, reduction of particle aggregation of the drug, absence of crystallinity, increased wettability and dispersibility, and alteration of surface properties of the drug particles may be responsible for the enhanced solubility and dissolution rate of gliclazide from its SDs and PMs. DSC of gliclazide SDs in PEG 4000 and corresponding PMs do not indicate the presence of crystalline gliclazide, because gliclazide dissolved completely below its melting point. The absence of an endothermic peak of gliclazide in the DSC thermograms of SDs with PEG 4000 shows the conversion of gliclazide from the crystalline to the microcrystalline state. The FTIR spectroscopic studies show the presence of intermolecular hydrogen bonding between gliclazide and PEG 4000 in the solid state. It can be concluded that gliclazide SDs with PEG 4000 provide a promising way to enhance its solubility and dissolution rate but are less effective than those prepared with PEG 6000.

\section{ACKNOWLEDGMENTS}

The authors are grateful to Aristo Pharmaceuticals Pvt. Ltd, Mumbai, India, and Clariant $\mathrm{GmbH}$, Sulzbach, Germany, for their interest and for supplying gliclazide and various grades of polyethylene glycol, respectively. Mr. S. Biswal acknowledges the Principal, Royal College of Pharmacy and Health Sciences, Berhampur, for providing the necessary facilities.

\section{REFERENCES}

1. Reynolds, J. E. F., Ed. Martindale: The Extra Pharmacopoiea XXX, 30th ed.; Pharmaceutical Press: London, 1993; pp 279-280.

2. Dollery, S. C., Ed. Therapeutic Drugs; Churchill Livingstone: London, 1991.

3. Harrower, A. D. Comparison of efficacy, secondary failure rate and complications of sulfonylurea. J. Diabetes Complicat. 1994, 8, 201-203.

4. Palmer, K. J.; Brogden, R. N. Gliclazide, an update of its pharmacological properties and therapeutic efficacy in NIDDM. Drugs 1993, 46, 92-125.

5. Ford, J. L. The current status of solid dispersions. Pharm. Acta Helv. 1986, 61, 69-88.

6. Özkan, Y.; Atay, T.; Díkman, N.; Isimer, A.; Aboul-Enein, Y. H. Improvement of water solubility and in vitro 
dissolution rate of gliclazide by complexation with $\beta$-cyclodextrin. Pharm. Acta Helv. 2000, 74, 365-370.

7. Winters, S.; York, P.; Timmins, P. Solid state examination of a gliclazide: beta-cyclodextrin complex. Eur. J. Pharm. Sci. 1997, 5, 209-214.

8. Sapkal, N. P.; Kilor, V. A.; Bhusari, K. P.; Daud, A. S. Evaluation of some Methods for preparation of gliclazide- $\beta$-cyclodextrin inclusion complexes. Trop. J. Pharm. Res. 2007, 6 (4), 833-840.

9. Aggarwal, S.; Singh, P. N.; Mishra, B. Studies on solubility and hypoglycemic activity of gliclazide beta-cyclodextrin-hydroxypropylmethylcellulose complexes. Pharmazie 2002, 57, 191-193.

10. Hong, S. S.; Lee, S. H.; Lee, Y. J.; Chung, S. J.; Lee, M. H.; Shim, C. K. Accelerated oral absorption of gliclazide in human subjects from a soft gelatin capsule containing a PEG 400 suspension of gliclazide. J. Controlled Release 1998, 51, 185-192.

11. Biswal, S.; Sahoo, J.; Murthy, P. N.; Giradkar, P. R.; Avari, J. G. Enhancement of dissolution rate of gliclazide using solid dispersions with polyethylene glycol 6000. AAPS PharmaSciTech 2008, 9 (2), 563-570.

12. Biswal, S.; Sahoo, J.; Murthy, P. N. Physicochemical Properties of Solid Dispersions of Gliclazide in PolyvinylpyrrolidoneK-90. AAPS PharmSciTech 2009, 10 (2), 329-334.

13. Higuchi, T.; Connors, K. Phase-solubility techniques. Adv. Anal. Chem. Instrum. 1965, 4, 117-123.

14. Nurten, O.; Sefika, O. Improvement of dissolution properties of furosemide by complexation with $\beta$-cyclodextrin. Drug Dev. Ind. Pharm. 1998, 24 (1), 19-25.

15. Ahmad, M.; Abdul, F.; Hridaya N. B. Preparation and in vitro evaluation of solid dispersions of halofantrine. Int. J. Pharm. 2002, 235, 17-33.

16. Trapani, G.; Franco, M.; Latrofa, A.; Pantaleo, M. R.; Provenzano, M. R.; Sanna, E.; Maciocco, E.; Liso, G. Physicochemical characterization and in vivo properties of Zolpidem in solid dispersions with polyethylene glycol 4000 and 6000. Int. J. Pharm. 1999, 184, 121-130.

17. Mura, P.; Manderioli, A.; Bramanti, G.; Ceccarelli, L. Properties of solid dispersions of naproxen in various polyethylene glycols. Drug Dev. Ind. Pharm. 1996, 22 (9-10), 909-916.

18. Khan, C. A.; Rhodes, C. T. The concept of dissolution efficiency. J. Pharm. Pharmacol. 1975, 27, 48-49.

19. Arias, M. J.; Gines, J. M.; Moyano, J. R.; Rabasco, A. M. Dissolution properties and in vivo behavior of triamterene in solid dispersions with polyethylene glycols. Pharm. Acta Helv. 1996, 71, 229-235.

20. Costa, P.; Lobo, J. M. S. Modeling and comparison of dissolution profiles. Eur. J. Pharm. Sci. 2001, 13, 123-133.

21. Barzegar-Jalali, M.; Maleki, N.; Garjani, A.; Khandar, A. A.; Haji- Hosseinloo, M.; Jabbari, R. C.; Dastmalchi,
S. Enhancement of dissolution rate and antiinflammatory effects of piroxicam using solvent deposition technique. Drug Dev. Ind. Pharm. 2002, 28, 681-686.

22. Mooter, G. V.; Augustijns, P.; Blaton, N.; Kinget, R. Physico-chemical characterization of solid dispersions of temazepam with polyethylene glycol 6000 and PVP K30. Int. J. Pharm. 1998, 164, 67-80.

23. Valizadeh, H.; Nokhodchi, A.; Qarakhani, N.; Zakeri-Milani, P.; Azarmi, S.; Hassanzadeh, D.; Lobenberg, R. Physicochemical characterization of solid dispersions of indomethacin with PEG 6000, Myrj 52, Lactose, Sorbitol, Dextrin, and Eudragit E100. Drug Dev. Ind. Pharm. 2004, 30 (3), 303-317.

24. Damian, F.; Blaton, N.; Naesens, L.; Balzarini, J.; Kinget, R.; Augustinjns, P.; Van den Mooter, G.

Physicochemical characterization of solid dispersions of the antiviral agent UC-781 with polyethylene glycol 6000 and Gelucire 44/14. Eur. J. Pharm. Sci. 2000, 10, 311-322.

25. Okonogi, S.; Yonemochi, E.; Oguchi, T.; Puttipipatkhachorn, S.; Yamamoto, K. Enhanced dissolution of ursodeoxycholic acid from the solid dispersion. Drug Dev. Ind. Pharm. 1997, 23 (11), 1115-1121.

26. Yamashita, K.; Nakate, T.; Okimoto, K.; Ohike, A.; Tokunaga, Y.; Ibuki, R.; Higaki, K.; Kimura, T. Establishment of new preparation method for solid dispersion formulation of tacrolimus. Int. J. Pharm. 2003, 267, 79-91.

\section{Dissolution Solutions Network}

The number one independent Pharma-Lab Blog site on The web with cutting edge information on USP Dissolution testing best suited for your marketing needs.

- Periodic commentaries by pharmaceutical scientists

- The most used collection of links in the dissolution Industry

- Trusted independent reviews

- Breaking pharmaceutical news

- 10 years of history on the internet

For a limited time only, the Dissolution Solutions Network is opening up advertising space at discount prices. Visit the Dissolution Solutions network. Stay informed, stay in touch, and stay current. www.dissolutionsolutions.net 